\title{
Editorial
}

\section{Can cardiomyocytes divide?}

There is no doubt that research in the field of myocardial regeneration has a remarkably exciting future. This is reflected by a recent statement of the special emphasis panel at the National Heart, Lung, and Blood Institute in the USA, which was assigned to identify areas which should be in the focus of cardiovascular research within the next decades. ${ }^{1}$ It says that we need to acquire " . . fundamental understanding of stem cell biology, cardiogenic differentiation, and cell cycle control". However, there are certain limitations we face when we want to embark on this field of research:

(1) the availability of human tissue necessary to study cardiomyocyte division is limited and the heart consists of a heterogenous cell population;

(2) compared to the contractile protein apparatus there is only a low abundance of most cell cycle regulating factors; (3) it may be necessary to convince people that counting cells alone may not give us the right answer about tissue regeneration.

If we wish to make progress in the field of myocardial regeneration in the nearer future, there are a number of issues that need to be addressed:

- which factors control cell cycle withdrawal in cardiomyocytes?

- what are their upstream regulators and what are their downstream targets?

- how do they interact with myogenic differentiation factors?

- how do they interact with hypertrophic and/or apoptotic signals in cardiomyocytes?

\section{Cytokinesis}

Cellular division is one of the key features and the basis of life in all metazoa. Therefore, any multicellular organ has to be endowed with a programme allowing cytokinesis. However, because of specific requirements some of these cells withdraw from the cell cycle during the perinatal period and become postmitotic. This is particularly true of cells comprising central organs such as the brain and the heart. For the latter, postnatal growth-which is essential to meet the increasing demand for work to support body growth - is achieved primarily by hypertrophy. This is probably because ongoing cellular division would render the heart mechanically unstable, and thus it would not be able to establish a functional circulatory homeostasis.

In contrast to skeletal muscle cells, where differentiation and cell division are mutually exclusive events, cardiac myocytes can contract and still divide; this is the case during fetal development, where the heart is responsible for providing oxygen rich blood to peripheral organs starting very early at embryogenesis, at a time where the heart itself is anything but fully differentiated. Thus, at an undifferentiated but already partially functional state cardiac cells can divide. This notion is in agreement with observations made in other organs which are known to retain a regenerative capacity throughout life. The liver, for example, has a regenerative capacity we have known about for a great many years, as reflected in the myth of Prometheus. Having stolen the secret of fire from the gods of the Olympus, Prometheus was condemned to having a portion of his liver eaten daily by an eagle. His liver regenerated overnight, thus providing the eagle with eternal food and Prometheus with eternal torture. However, not until recently have we realised that in order to divide even a liver cell has to retrodifferentiate. ${ }^{2}$

Several decades ago the German pathologist Linzbach postulated that the increase in the size of the failing heart could not be accounted for solely on the basis of hypertrophic growth. ${ }^{3}$ However, he was unable to demonstrate cardiomyocyte mitosis. This may have been because of the limited technology available at that time. In modern times there are reports which claim to detect increased cardiomyocyte division in the failing ${ }^{4}$ and infarcted ${ }^{5}$ heart. Despite the availability of improved technology, these studies are flawed by invalid underlying hypotheses. Here, the authors calculate the number of mitotic cardiomyocytes in healthy and diseased failing myocardium on the basis of two incorrect assumptions: (1) the left ventricle contains $5.8 \times 10^{9}$ myocytes; and (2) the duration of mitosis lasts less than one hour and equals length of cell division. According to these assumptions the authors conclude that $0.001 \%$ of cardiomyocytes are in mitosis at a given time point, while there is an increase in mitosis in the failing myocardium by the order of one magnitude, ${ }^{4}$ and by the order of nearly two magnitudes in the infarcted myocardium. ${ }^{5}$

To my knowledge there is no precedent for the assumption that any of our internal organs have a fixed number of cells as stated by these authors to be the case with the heart. Body size, sex, and age certainly contribute to a high variability among different individuals in regard to the number of cells in an organ like the heart. Secondly, the duration of mitosis may vary from cell to cell with organs exhibiting a high proliferation rate, where mitosis may take place within only 30 minutes, and organs with a rather slow proliferation rate, where mitosis may take place within 1-2 hours. Moreover, the extrapolation of the length of mitosis to the duration of cell division lacks any reasonable scientific basis. While the length of mitosis may vary by only a few hours between different cell types, the variation between the length of cell division may be several days as a result of the difference in the duration of G1 and G2 phase. If the analysis of the authors was correct, clinicians would encounter cardiac tumours much more frequently. However, primary myocardial tumours are rarely observed in adults, and if so are not of cardiomyocyte origin. Also, it is evident that functionally significant myocardial regeneration has not been documented in diseases and/or injuries that result in cardiomyocyte loss. So even if we accept the assumption that the adult heart is comprised of cardiomyocytes which have retained some degree of regenerative 
growth, cardiomyocyte division appears to be such an unfrequent event that you do not have to be a cardiologist to know that after injury our heart is not able to rejuvenate.

\section{Molecular mechanisms}

Despite the fact, that this field of research has gained quite an impetus within the last few years, cell cycle control in cardiomyocytes remains enigmatic, if one ponders upon the potential molecular mechanisms accounting for the essentially irreversible cell cycle withdrawal of cardiomyocytes:

- the loss of growth factor receptors or signalling components-however, most growth regulating cascades persist into adulthood

- the loss of cell cycle proteins-however, most cell cycle proteins exist or can be reinduced in adult cardiomyocytes

- the elaboration of antiproliferative factors-however, animal models with disruption of candidate genes are not characterised by an overt cardiac phenotype

- the antiproliferative effect of myogenic determination factors-however, in cardiomyocytes differentiation and proliferation are not mutually exclusive events.

Does it look like this is going to be a hopeless endeavour? Certainly not! As outlined at the beginning of this article, mammalian cardiomyocytes are endowed with the full cell cycle programme, and although normal adult cardiomyocytes have down regulated levels of cell cycle factors, they retain the ability to re-express these factors during hypertrophic growth. ${ }^{6}$ Thus, the critical question is not "Can cardiomyocytes divide?", rather it is "Why do cardiomyocytes not divide all the time?". With this modified question in mind we will progress much more quickly, because it implies that cardiomyocyte division is constantly suppressed through an active process. Thus, we may be able to induce cardiomyocyte division just by simply switching off this suppressive mechanism. This concept is fostered by the observation that cardiomyocyte cell cycle re-entry can easily be achieved by forced ectopic expression of cell cycle activators, ${ }^{7}$ or by removing inhibitory cytoplasm from cardiomyocyte nuclei. ${ }^{8}$

How can we identify these switches which are responsible for the suppression of cardiomyocyte division? Again, there is a helping hand called programmed cell death or apoptosis. In general, cell death and cell cycle are tightly linked programmes which have to be kept in a fine tuned equilibrium. ${ }^{9}$ A tight interplay between apoptotic signalling and cell cycle control has been described for a variety of different cell types, though there is still a lack of evidence in cardiomyocytes. According to this notion, the same factors should be responsible for the suppression of cell cycle and apoptosis. This is meaningful in a teleological sense, since a cell which is not allowed to divide should also be not allowed to die in order to preserve cellular homeostasis in a postmitotic organ such as the heart. Thus, studying antiapoptotic signalling pathways most likely will lead to the identification of cell cycle checkpoints in cardiomyocytes. But beware-again the significance of apoptosis will not be recognised just by counting dead cells.

In conclusion, the initial question "Can cardiomyocytes divide?" can be answered with "Yes, they can!". However, at no time is the healthy or diseased human heart able to replace damaged tissue efficiently. The much more appropriate question "Why do cardiomyocytes not divide all the time?", I have to answer with "We do not know yet!". Nevertheless, searching for the molecular basis of cell cycle withdrawal and apoptosis in cardiomyocytes, rather than counting mitotic figures or dead cells, should provide us with critical information required to initiate interventions aimed at myocardial regeneration in the not too distant future.

RÜDIGER VON HARSDORF

Universitätsklinikum Charité

Medizinische Klinik m S Kardiologie

Augustenburger Platz 1

13353 Berlin, Germany

ruediger.harsdorf@charite.de

National Heart, Lung, and Blood Institute. Special emphasis panel at the NHLB Institute, USA. Circulation 1997;95:766-70.

Michalopoulos GK, DeFrances MC. Liver regeneration. Science 1997;276: $60-5$

3 Linzbach AJ. Pathogenese der Herzinsuffizienz bei Hypertonie. Triangel 1975;14:17-26.

4 Kajstura J, Leri A, Finato N, et al. Myocyte proliferation in end-stage cardiac failure in humans. Proc Natl Acad Sci, USA 1998;95:8801-5.

5 Beltrami AP, Urbanek K, Kajstura J, et al. Evidence that human cardiac Beltrami AP, Urbanek K, Kajstura J, et al. Evidence that human cardiac
myocytes divide after myocardial infarction. N Engl f Med 2001;344:17507 .

$6 \mathrm{Li}$ JM, Poolman RA, Brooks G. Role of G1 phase cyclins and cyclin-dependent kinases during cardiomyocyte hypertrophic growth in rats. Am $\mathcal{F}$ Physiol 1998;275:H814-22.

7 von Harsdorf R, Hauck L, Mehrhof F, et al. E2F-1 overexpression in cardiomyocytes induces downregulation of $\mathrm{p} 21 \mathrm{CIP} 1$ and $\mathrm{p} 27 \mathrm{KIP} 1$ and release of active cyclin-dependent kinases in the presence of insulin-like growth factor-I. Circ Res 1999;85:128-36.

8 Engel FB, Hauck L, Cardoso MC, et al. A mammalian myocardial cell-free system to study cell cycle reentry in terminally differentiated cardiomyocytes. Circ Res 1999;85:294-301.

9 King KL, Cidlowski JA. Cell cycle regulation and apoptosis. Annu Rev Physiol 1998;60:601-17. 